\title{
On the Concavity of the Consumption Function with a Quadratic Utility under Liquidity Constraints
}

\author{
Shin-Ichi Nishiyama ${ }^{1}$, Ryo Kato ${ }^{2}$ \\ ${ }^{1}$ Tohoku University, Sendai, Japan \\ ${ }^{2}$ Bank of Japan, Tokyo, Japan \\ Email: nishiyama@econ.tohoku.ac.jp,ryou.katou@boj.or.jp
}

Received April 26, 2012; revised May 28, 2012; accepted June 3, 2012

\begin{abstract}
This paper demonstrates the concavity of the consumption function of infinitely living households under liquidity constraints who are not prudent-i.e. with a quadratic utility. The concavity of the consumption function is closely related to the 3-convexity of the value function.
\end{abstract}

Keywords: Consumption Function, Liquidity Constraints

\section{Introduction}

Since the numerical illustration by Deaton [1], researchers have been aware that liquidity constraints generate a concavity in the consumption function. However, analytics of the concavity due to liquidity constraints has remained unknown until recently. Carroll and Kimball [2] made the first important attempt in setting an analytical foundation and showed the concavity of the consumption function when the consumer's optimization has a finite horizon. Technically, they exploit the convexity of marginal value function in the terminal period and use the backward induction to show the convexity of marginal value function in the current period. However, in the context of infinite horizon, this particular approach is not applicable since the terminal period's value function is not defined.

In this paper, we offer an analytical foundation of the concavity of the consumption function in the context of infinite horizon, when consumer's utility is quadratic. Taking a different approach to Carroll and Kimball [2], we directly prove the 3-convexity or Levinson's Inequality (Levinson [3]) of the value function and show that the consumption function is concave. The concept of 3-convexity is extremely convenient when characterizing the value function, especially with the infinite horizon. Thus, we regard this 3-convexity approach as a complement, rather than a substitute, to Carroll and Kimball's [2] backward induction approach in the finite horizon setting.

Finally, it should be emphasized that under the model that we consider-i.e. consumer's utility is quadraticthe concavity is not generated by prudence of the con- sumer, but is solely generated by the presence of liquidity constraints ${ }^{1}$. By the virtue of this set-up, we can solely focus on the analytical mechanism how liquidity constraints generate the concavity in the consumption function. The rest of the paper is organized as follows. Sectiones 2 discuss the set-up of the model. Section 3 clarifies the concept of 3-convexity, shows the 3-convexity of the value function and proves the concavity of the consumption function. Section 4 provides some concluding remarks.

\section{The Model}

We assume a very simple infinite horizon dynamic optimization problem where consumer's utility is quadratic and time-separable. Further, consumer faces no uncertainty in terms of rate-of-return on the net wealth and in terms of labor income. The only source that makes the dynamic optimization problem non-standard is the existence of liquidity constraints - the net wealth cannot be negative. Thus, consumer's dynamic optimization problem can be formulated as follows.

$$
\begin{gathered}
V_{t}\left(w_{t}\right)=\max _{c_{t+i}} \sum_{i=0}^{\infty} \beta^{i} U\left(c_{t+i}\right) \\
\text { s.t. } w_{t+i+1}=R \times\left(w_{t+i}-c_{t+i}\right)+y \\
w_{t+i+1} \geq 0
\end{gathered}
$$

where $c_{t}$ stands for consumption, which is the control variable of the consumer, and $w_{t}$ stands for the net wealth, which is the state variable of the optimization problem.

${ }^{1}$ For the concavity of the consumption function generated by prudence, see Carroll and Kimball [4]. 
Period-by-period utility is defined as a quadratic function in consumption, i.e. $U\left(c_{t}\right)=a c_{t}-(b / 2) c_{t}^{2}$, where $a$ and $b$ are positive constant parameters. Discount rate $\beta$, interest rate $R$, and labor income $y$, are assumed to be time-invariant ${ }^{2}$. Recursive nature of this infinite horizon problem allows us to reduce it into the following Bellman equation:

$$
\begin{gathered}
V(w)=\max _{c \leq w+y / R}\left\{a c-\frac{b}{2} c^{2}+\beta V(\tilde{w})\right\} \\
\text { s.t. } \tilde{w}=R \times(w-c)+y
\end{gathered}
$$

It should be noted that since the optimization horizon is infinite, the value functions in the subsequent period will converge to a certain function $V(\cdot)$ as can be seen on both sides of the Bellman Equation (2). Furthermore, this converged value function is a consequence of recursive optimization under liquidity constraints from the future period and therefore should be distinguished from the value function under liquidity unconstrained case $^{3}$. In other words, the value function under liquidity constraints will no longer be a quadratic function even under quadratic utility, which is in sharp contrast to the case without liquidity constraint whose value function is, of course, quadratic.

Taking the first-order condition of Equation (2) with respect to consumption will yield the following equation:

$$
c(w)=\frac{a}{b}-\frac{\beta}{b} R V^{\prime}(\tilde{w})
$$

The function $c(w)$ on the left-hand side characterizes the optimal consumption as a function of the current net wealth. Further, by invoking the envelope theorem (or Benveniste-Scheinkman formula) on Equation (2), we can derive the following relation between the current shadow price of the net wealth-i.e. the marginal value function evaluated at the current net wealth-and the future shadow price.

$$
V^{\prime}(w)=\beta R V^{\prime}(\tilde{w})
$$

Combining Equations (3) and (4), we obtain the following key equation:

$$
c(w)=\frac{a}{b}-\frac{1}{b} V^{\prime}(w)
$$

\footnotetext{
${ }^{2}$ Uncertainty in rate-of-return or labor income can be easily introduced in the model set-up, but will not alter the main implication. Indeed, since the very message of this paper is the concavity of consumption function under deterministic environment, introduction of uncertainty will only obscure the main point of this paper.

${ }^{3} \mathrm{We}$ assume the regularity condition on discount rate to ensure the Contraction Mapping Theorem to hold. For more rigorous treatment on this issue, see for Stokey and Lucas [5]. Also, for the existence of converged value function under the case where control variables are constrained, see for instance Chmielewski and Manousiouthakis [6].

${ }^{4}$ It should be noted that when the value function is quadratic (as in the case when preference is quadratic and without liquidity constraints), the optimal consumption function will be linear. This can be easily seen from Equation (5).
}

The virtue of Equation (5) is that it relates the current optimal consumption to the current shadow price of the net wealth rather than the future shadow price of the net wealth as in Equation (3). This key relationship enables us to infer the characteristics of the optimal consumption function by investigating the nature of the marginal value function. Or putting it another way, it suffices to characterize the marginal value function in order to characterize the optimal consumption function ${ }^{4}$.

Some remarks are in order. If the value function is three times differentiable, then 3-convexity of the value function is equivalent to positiveness of the third derivative of the value function-i.e., $V^{\prime \prime \prime}(\cdot) \geq 0$. However, as pointed out by Carroll and Kimball [2], the marginal value function in Equation (5) is "kinked" under the deterministic environment with liquidity constraints that $V^{\prime \prime \prime}(\cdot)$ is not well defined. Thus, it is not appropriate to rely on third order differentiability of $V(\cdot)$ in proving the concavity of the consumption function, especially in our case of consideration. Fortunately, the concept of 3-convexity is more general in its applicability. As long as the value function is once differentiable, 3-convexity implies the convexity of marginal value function even if the marginal value function is kinked and this implication does not require the function to be 2 or 3 times differentiable. It is this property of 3-convexity that makes it relatively easy to show the concavity of the consumption function in the presence of liquidity constraints. The following section clarifies the concept of 3-convexity and then shows that the value function is 3-convex.

\section{Main Results}

We first define the notion of 3-convexity.

Definition 1 (3-convexity). A function $f:[a, b] \rightarrow \mathfrak{R}$ is said to be 3-convex on $[a, b]$ if for $\forall x_{1}, x_{2} \in[a, b]$ such that $x_{1} \neq x_{2}$

$$
\begin{gathered}
\underbrace{f\left(\frac{x_{1}+x_{2}}{2}\right)-\left[\frac{1}{2} f\left(x_{1}\right)+\frac{1}{2} f\left(x_{2}\right)\right]}_{A} . \\
\geq \underbrace{f\left(x_{2}\right)-\left[\frac{1}{2} f\left(\frac{x_{1}+x_{2}}{2}\right)+\frac{1}{2} f\left(\frac{3 x_{2}-x_{1}}{2}\right)\right]}_{B} .
\end{gathered}
$$

The inequality (6) is a special case of Levinson's inequality (Levinson [3]) which can be regarded as a higher-order Jensen's inequality. As Jensen's inequality is closely related with the notion of convexity, so is Levinson's inequality with 3-convexity. The intuition of the inequality (6) can be vividly captured by Figure 1. The left-hand side of the inequality (i.e. denoted $A$ ) represents the difference between the value of function 


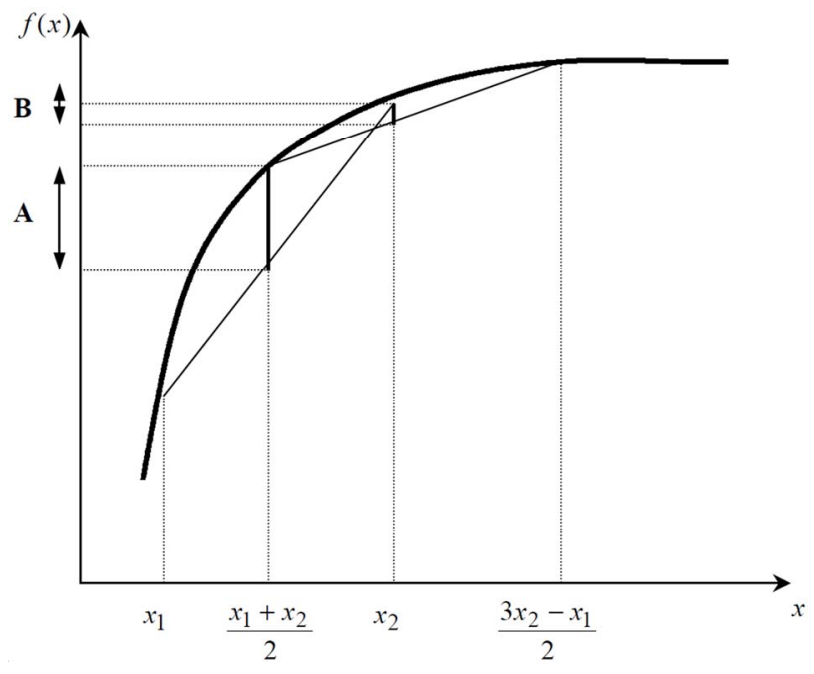

Figure 1. Illustration of 3-convexity.

evaluated at the mid-point of $x_{1}$ and $x_{2}$ to the midpoint of the chord from $x_{1}$ and $x_{2}$. It is possible to interpret $A$ as a magnitude of concavity of a function in the domain $\left[x_{1}, x_{2}\right]$. The right-hand side of the inequality (i.e. denoted $B$ ) can be interpreted in the similar fashion with a difference that domain is now

$$
\left[\frac{x_{1}+x_{2}}{2}, \frac{3 x_{2}-x_{1}}{2}\right] \text {. }
$$

Thus, intuitively speaking, the function will be 3-convex if the magnitude of concavity decreases as $X$ increases $^{5}$.

Next, we state the lemma that links 3-convexity of the function to convexity of the marginal function. The following lemma is a special case of the more general theorem that links $n$-convexity to convexity of $(n-k)$ th derivative of a function. Rigorous proof of the theorem is well beyond the scope of this paper and will be omitted.

Lemma 1. If a function $f: \mathfrak{R} \rightarrow \mathfrak{R}$ is 3-convex on $[a, b]$, then the first derivative $f^{\prime}: \mathfrak{R} \rightarrow \mathfrak{R}$ exists and is convex on $[a, b]$.

Proof. See Pecaric et al. [7].

We are now in the position to state the key theorem of this paper.

Theorem 1. Let $V(w)$ be the value function stated in (2). Then for any $w \in(0, \infty), V(w)$ is 3-convex.

Proof. Let $w_{1}$ and $w_{2}$ be some arbitrary number in $[0, \infty)$ such that $w_{1}<w_{2}$. Then it suffices to show the following inequality:

$$
\begin{aligned}
& V\left(\frac{w_{1}+w_{2}}{2}\right)-\left[\frac{1}{2} V\left(w_{1}\right)+\frac{1}{2} V\left(w_{2}\right)\right] \\
& \geq V\left(w_{2}\right)-\left[\frac{1}{2} V\left(\frac{w_{1}+w_{2}}{2}\right)+\frac{1}{2} V\left(\frac{3 w_{2}-w_{1}}{2}\right)\right]
\end{aligned}
$$

which is equivalent in showing that

${ }^{5}$ Or, in the continuous analouge, $f^{\prime \prime}(x)$ is increasing in $x$.

$$
\begin{aligned}
& 3 V\left(\frac{w_{1}+w_{2}}{2}\right)+V\left(\frac{3 w_{2}-w_{1}}{2}\right) \\
& -V\left(w_{1}\right)-3 V\left(w_{2}\right) \geq 0 .
\end{aligned}
$$

Let sequence $\left\{c_{1, t+j}\right\}_{j=0}^{\infty}$ and $\left\{c_{2, t+j}\right\}_{j=0}^{\infty}$ be the optimal consumption path given state $w_{1}$ and $w_{2}$, respectively. Now, define

$$
\bar{c}_{t+j}=\frac{1}{2} c_{1, t+j}+\frac{1}{2} c_{2, t+j} \text { and } \hat{c}_{t+j}=\frac{3}{2} c_{2, t+j}-\frac{1}{2} c_{1, t+j} .
$$

Further, define

$$
\bar{w}=\frac{1}{2} w_{1}+\frac{1}{2} w_{2} \text { and } \hat{w}=\frac{3}{2} w_{2}-\frac{1}{2} w_{1} .
$$

Then from Chmielewski and Manousiouthakis [6], the sequence

$$
\left.\left\{\bar{c}_{t+j}\right\}_{j=0}^{\infty} \text { (or }\left\{\hat{c}_{t+j}\right\}_{j=0}^{\infty}\right)
$$

is feasible, but not necessarily equal to the optimal consumption path given the state $\bar{w}$ (or $\hat{w}$ ). Therefore,

$$
V(\bar{w}) \geq \sum_{j=0}^{\infty} \beta^{j} U\left(\bar{c}_{t+j}\right) \text { and } V(\hat{w}) \geq \sum_{j=0}^{\infty} \beta^{j} U\left(\hat{c}_{t+j}\right) \text {. }
$$

Then from the inequality (7), it follows that

$$
\begin{aligned}
& 3 V(\bar{w})+V(\hat{w})-V\left(w_{1}\right)-3 V\left(w_{2}\right) \\
\geq & 3 \sum_{j=0}^{\infty} \beta^{j} U\left(\bar{c}_{t+j}\right)+\sum_{j=0}^{\infty} \beta^{j} U\left(\hat{c}_{t+j}\right) \\
& -\sum_{j=0}^{\infty} \beta^{j} U\left(c_{1, t+j}\right)-3 \sum_{j=0}^{\infty} \beta^{j} U\left(c_{2, t+j}\right) .
\end{aligned}
$$

Rearranging the right-hand side of the inequality (8) and from the definition of the utility function, it follows that

$$
\begin{aligned}
R H S= & \sum_{j=0}^{\infty} \beta^{j}\left[3\left(a \bar{c}_{t+j}-\frac{b}{2} \bar{c}_{t+j}^{2}\right)+\left(a \hat{c}_{t+j}-\frac{b}{2} \hat{c}_{t+j}^{2}\right)\right] \\
& -\sum_{j=0}^{\infty} \beta^{j}\left[\left(a c_{1, t+j}-\frac{b}{2} c_{1, t+j}^{2}\right)+3\left(a c_{2, t+j}-\frac{b}{2} c_{2, t+j}^{2}\right)\right] \\
= & \sum_{j=0}^{\infty} \beta^{j}\left[a\left(c_{1, t+j}+3 c_{2, t+j}\right)-\frac{b}{2}\left(c_{1, t+j}^{2}+3 c_{2, t+j}^{2}\right)\right] \\
& -\sum_{j=0}^{\infty} \beta^{j}\left[a\left(c_{1, t+j}+3 c_{2, t+j}\right)-\frac{b}{2}\left(c_{1, t+j}^{2}+3 c_{2, t+j}^{2}\right)\right]=0
\end{aligned}
$$

Thus, $3 V(\bar{w})+V(\hat{w})-V\left(w_{1}\right)-3 V\left(w_{2}\right) \geq 0$. This proves the theorem.

The concavity of the optimal consumption function follows naturally from Lemma 1 and Theorem 1 .

Theorem 2. Let $c(w)$ be the optimal consumption function of the dynamic optimization problem (2). Then for any $w$ in $[0, \infty), c(w)$ is concave.

Proof. Let $w_{1}$ and $w_{2}$ be some arbitrary number in $[0, \infty)$ such that $w_{1}<w_{2}$. Then it suffices to show, 


$$
c\left(\lambda w_{1}+(1-\lambda) w_{2}\right) \geq \lambda c\left(w_{1}\right)+(1-\lambda) c\left(w_{2}\right)
$$

where $0<\lambda<1$. From Equation (5), this is equivalent in showing that

$$
V^{\prime}\left(\lambda w_{1}+(1-\lambda) w_{2}\right) \leq \lambda V^{\prime}\left(w_{1}\right)+(1-\lambda) V^{\prime}\left(w_{2}\right) .
$$

Now from Theorem $1, V(w)$ is 3-convex, which in turn implies that $V^{\prime}(w)$ is convex from Lemma 1 . This proves the theorem.

\section{Concluding Remark}

This paper showed, in the context of infinite horizon, how the presence of liquidity constraints generate a concavity in the consumption function, even when consumer is not prudent-i.e. preference is quadratic. In showing the concavity of the consumption function, we directly proved the 3-convexity (also known as Levinson's inequality [3]) of the value function. This direct approach utilizing 3-convexity of the value function is convenient in characterizing the consumption function, especially in the infinite horizon context and can thought to be a complement, rather than a substitute, to Carroll and Kimball's [2] backward induction approach for the finite horizon.

We thank Hiroshi Fujiki, Keiko Murata, Makoto Saito and, especially, Miles Kimball for their helpful comments and suggestions. This paper was written while the first author was at the Institute for Monetary and Economic Studies, Bank of Japan. The views expressed in this paper are those of the authors and do not necessarily reflect those of the Bank of Japan or the Institute for Monetary and Economic Studies.

\section{REFERENCES}

[1] A. Deaton, "Saving and Liquidity Constraints," Econometrica, Vol. 59, No. 5, 1991, pp. 1221-1248. doi:10.2307/2938366

[2] C. D. Carroll and M. S. Kimball, "Liquidity Constraints and Precautionary Saving,” NBER Working Paper 8496, National Bureau of Economic Research, Inc., 2001.

[3] N. Levinson, "Generalization of an Inequality of Ky Fan,” Journal of Mathematical Analysis and Applications, 8, 1964, pp. 133-134. doi:10.1016/0022-247X(64)90089-7

[4] C. D. Carroll and M. S. Kimball, "On the Concavity of the Consumption Function,” Econometrica, Vol. 64, No. 4, 1996, pp. 981-992. doi:10.2307/2171853

[5] N. L. Stokey, R. E. Lucas Jr. and E. C. Prescott, "Recursive Methods in Economic Dynamics," Harvard University Press, Cambridge, 1989.

[6] D. Chmielewski and V. Manousiouthakis, "On Constrained Infinite-time Linear Quadratic Optimal Control, Systems and Control Letters, Vol. 29, No. 3, 1996, pp. 121-129. doi:10.1016/S0167-6911(96)00057-6

[7] J. E. Pecaric, F. Proschan and Y. L. Tong, "Convex Functions, Partial Orderings, and Statistical Applications Academic Press, San Diego, 1992. 